\title{
Glucose-lowering effects of 7-day treatment with SGLT2 inhibitor confirmed by intermittently scanned continuous glucose monitoring in outpatients with type 1 diabetes. A pilot study
}

\author{
Akira Kurozumi, Yosuke Okada and Yoshiya Tanaka
}

\begin{abstract}
First Department of Internal Medicine, School of Medicine, University of Occupational and Environmental Health, Japan, Kitakyushu 807-8555, Japan
\end{abstract}

\begin{abstract}
The present study used intermittently scanned continuous glucose monitoring (isCGM) in 10 patients with type 1 diabetes mellitus (T1DM) to evaluate the efficacy and safety of 7-day outpatient treatment with the combination of intensive insulin therapy and sodium-glucose transporter 2 inhibitor (SGLT2-I). All participants wore isCGM and were treated with either $50 \mathrm{mg}$ /day ipragliflozin or $5 \mathrm{mg} /$ day dapagliflozin. The primary outcome, percent time with glucose at $70-180 \mathrm{mg} / \mathrm{dL}$ (TIR: time in range), improved significantly following the addition of SGLT2-I $(p=0.005)$. TIR increased from $36.0 \%$ before addition of SGLT2-I to $70.7 \%$ on day 7 . Although none of the patients achieved TIR of $70 \%$ or higher before the addition of SGLT2-I, 6 patients met that criteria TIR on day 7. The secondary outcome measures, standard deviation (SD) of glucose, average plasma glucose, percent time with glucose at $>180 \mathrm{mg} / \mathrm{dL}$ (TAR: time above range), maximum plasma glucose, high blood glucose index (HBGI) and average nocturnal plasma glucose (midnight to 05:59 AM) detected by isCGM, also improved significantly by SGLT2-I. There were no significant differences in percent time with glucose at $<70 \mathrm{mg} / \mathrm{dL}$ (TBR: time below range), minimum plasma glucose and low blood glucose index (LBGI). Our results using isCGM in an actual clinical setting showed that 7-day use of SGLT2-I with intensive insulin therapy improved plasma glucose fluctuations and mean plasma glucose levels without inducing hypoglycemia in patients with T1DM.
\end{abstract}

Key words: Type 1 diabetes mellitus, Intermittently scanned continuous glucose monitoring, Sodium glucose cotransporter-2 inhibitor

WHILE data from the Japan Diabetes Clinical Data Management Study Group (JDDM) in 2018 showed improvement in glycated hemoglobin (HbA1c) levels over time in Japanese patients with type 1 diabetes mellitus (T1DM) following the development of new insulin preparations, the use of these forms of preparation have been linked to a gradual increase in the body mass index (BMI) in both patients with T1DM and type 2 diabetes mellitus (T2DM) as well as the number of obese patients (JDDM in 2018). Several clinical trials have been conducted to define the indications for use of sodiumglucose transporter 2 inhibitor (SGLT2-I) in T1DM [1-3]. A meta-analysis of 14 such randomized controlled trials

Submitted Sep. 10, 2020; Accepted Oct. 27, 2020 as EJ20-0577 Released online in J-STAGE as advance publication Nov. 18, 2020 Correspondence to: Akira Kurozumi, MD, PhD, First Department of Internal Medicine, School of Medicine, University of Occupational and Environmental Health, Japan, 1-1 Iseigaoka, Yahatanishi-ku, Kitakyushu 807-8555, Japan.

E-mail: akira@med.uoeh-u.ac.jp including three phase III clinical trials; the Dapagliflozin Evaluation in Patients with Inadequately Controlled Type 1 Diabetes (DEPICT-1) trial [1], Empagliflozin as Adjunctive to Insulin Therapy in Type 1 Diabetes (The EASE Trials) [2], and the in Tandem trial of sotagliflozin [3], which is not marketed in Japan, showed that SGLT2I reduces $\mathrm{HbA} 1 \mathrm{c}$ levels by $0.4 \%$ compared with placebo, fasting plasma glucose level by $21 \mathrm{mg} / \mathrm{dL}$, systolic blood pressure by $3.4 \mathrm{mmHg}$, daily insulin dose by 6 units and body weight by $2.7 \mathrm{~kg}$ [4]. In Japan, some use of SGLT2-I has been covered by the National Health Insurance for T1DM since December 2018.

In 2019, the international panel of the Advanced Technologies and Treatments for Diabetes (ATTD) Congress issued treatment guidelines for glycemic control targets under continuous glucose monitoring (CGM) [5]. According to these guidelines, the target therapeutic range is a blood glucose level of 70-180 $\mathrm{mg} / \mathrm{dL}$ for patients, excluding pregnant women. The time in range (TIR) recorded through the CGM is defined as 
the percent time spent at blood glucose level within the above range, while the time below range (TBR) is defined as the percent time spent at blood glucose level in the hypoglycemic range $(<70 \mathrm{mg} / \mathrm{dL})$, and the time above range (TAR) represents the percent time spent at blood glucose level in the hyperglycemic range $(>180$ $\mathrm{mg} / \mathrm{dL})$. A TIR of $70 \%$ or higher is preferred even in patients with T1DM. The TIR is associated with HbA1c levels and diabetic complications [6], and a recent metaanalysis study of 18 CGM-based studies concluded that TIR levels correlate well with those of HbA1c [7].

Only a few studies have evaluated the effects of SGLT2-I in Japanese patients with T1DM using intermittently scanned continuous glucose monitoring (isCGM), especially those treated at the outpatient clinics. The present study used isCGM to evaluate the early efficacy and safety of the combination of intensive insulin therapy and SGLT2-I in an actual clinical setting in 10 patients with T1DM treated at the outpatient clinic.

\section{Subjects and Methods}

\section{Participants}

The study participants were 10 patients with T1DM (acute type: 4 patients, slowly progressive type: 5 patients, fulminant type: 1 patient), who visited the outpatient clinic of the Department of Endocrinology, Metabolism and Diabetes, University of Occupational and Environmental Health, Japan. We excluded patients treated with SGLT2-I and those with severe liver dysfunction (aspartate aminotransferase $>100 \mathrm{IU} / \mathrm{L}$ ), estimated glomerular filtration rate (eGFR) of $<30 \mathrm{~mL} / \mathrm{min} /$ $1.73 \mathrm{~m}^{2}$, BMI $<18.0 \mathrm{~kg} / \mathrm{m}^{2}$ and those with history of allergy to SGLT2-I. Although there were no restrictions on the use of oral anti-hyperglycemic agents other than SGLT2-I, changes of drugs and insulin dose were prohibited while wearing the isCGM. The study was approved by the Ethics Committee of the University of Occupational and Environmental Health, Japan (\#H27-186).

\section{Study design}

This study was a retrospective study. All participants wore isCGM (FreeStyle Librepro ${ }^{\circledR}$, Abbott) and the data used for analysis were obtained from day 3 of wearing isCGM to ensure stability of the data. No changes were made to diet or exercise therapy or drugs and insulin dose (except the SGLT2-I). We separated the isCGM data into baseline data (those recorded before treatment from 0:00 on day 3 to $23: 59$ on day 3 of wearing the is CGM), and those of post-treatment [after treatment with either $50 \mathrm{mg}$ /day ipragliflozin (IPR) $(n=6)$ or 5 $\mathrm{mg}$ /day dapagliflozin (DAPA) $(n=4)$ recorded over the period from 0:00 on day 4 of wearing the isCGM for 7 days). The patients underwent in-hospital education program designed to prevent euglycemic ketoacidosis. The program included advice on drinking sufficient amount of water during the day, avoidance of excess carbohydrate restriction and, information on polydipsia, and detailed side-effects of treatment with SGLT2-I especially when the patients are feeling sick or lack appetite.

\section{Laboratory tests}

Blood samples were collected early in the morning after at least $12-\mathrm{h}$ fasting, through a venous line placed in the median vein using an indwelling catheter. Measurement of each parameter was outsourced to SRL Co. (Tokyo, Japan) using standard methods. All samples were stored at $-80^{\circ} \mathrm{C}$ until measurement.

\section{Study outcomes}

The primary outcome was percent time at glucose between 70 and $180 \mathrm{mg} / \mathrm{dL}$ (TIR) detected by isCGM. The secondary outcomes were standard deviation (SD) of glucose, average plasma glucose, percent time with glucose at $<70 \mathrm{mg} / \mathrm{dL}$ (TBR), percent time with glucose at $>180 \mathrm{mg} / \mathrm{dL}$ (TAR), maximum plasma glucose, minimum plasma glucose, high blood glucose index (HBGI), low blood glucose index (LBGI), \%coefficient of variation $(\% \mathrm{CV}), \mathrm{SD}$ of glucose during nocturnal period (0:00-05:59) and average plasma glucose during nocturnal period (0:00-05:59) detected by the isCGM. We also defined the glycemic control achieved group as those patients who achieved TIR of $\geq 70 \%$ on day 7 .

\section{Statistical analysis}

Data were expressed as mean \pm SD. Friedman test was used for comparison of the baseline and post-treatment data. The time-course changes in each glucose parameter detected by the isCGM were compared by the Dunnett test (data of Table 2). The spearman correlation was used for analysis of the data listed in Table 3. The MannWhitney $U$ test was used to compare the achievement and non-achievement groups in Table 4 (achievement group: patients who showed improvement in percent time with glucose at $70-180 \mathrm{mg} / \mathrm{dL}$ to $\geq 70 \%$ after the combination treatment). The level of statistical significance was set at $p<0.05$. All statistical analyses were conducted using the Statistical Package for Social association version 21.0 (SPSS Inc., Chicago, IL).

\section{Results}

\section{Baseline characteristics}

Table 1 shows the baseline characteristics of the patients. The median age of the 10 participants was 59.5 
Table 1 Baseline characteristics of patients

\begin{tabular}{|c|c|}
\hline Sex (male:female) & $10(4: 6)$ \\
\hline Age (year) & $59.5(44.8-62.3)$ \\
\hline Diabetes duration (years) & $5.0(2.0-8.3)$ \\
\hline Body weight $(\mathrm{kg})$ & $61.3(53.0-68.5)$ \\
\hline Body mass index $\left(\mathrm{kg} / \mathrm{m}^{2}\right)$ & $22.7(21.0-27.1)$ \\
\hline $\mathrm{HbAlc}(\%)$ & $9.1(8.8-9.2)$ \\
\hline $\mathrm{FPG}(\mathrm{mg} / \mathrm{dL})$ & $101.5(81.5-188.0)$ \\
\hline AST (IU/L) & $22.5(18.0-30.5)$ \\
\hline ALT (IU/L) & $18.5(11.0-24.8)$ \\
\hline$\gamma$-GTP (IU/L) & $20.0(11.0-53.0)$ \\
\hline eGFR $\left(\mathrm{mL} / \mathrm{min} / 1.73 \mathrm{~m}^{2}\right)$ & $77.7(70.2-90.3)$ \\
\hline LDL-C (mg/dL) & $81.0(66.5-94.8)$ \\
\hline HDL-C (mg/dL) & $57.0(49.0-68.3)$ \\
\hline $\mathrm{TG}(\mathrm{mg} / \mathrm{dL})$ & $111.0(71.0-195.5)$ \\
\hline Required insulin dose (u/day) & $\begin{array}{l}\text { Total: } 34.0(22.8-57.0) \\
\text { Bolus: } 16.0(12.0-19.5) \\
\text { Basal: } 17.0(9.3-34.0)\end{array}$ \\
\hline Diabetic microangiopathy & $\begin{array}{l}\text { neuropathy: } 2 / 10 \\
\text { retinopathy: } 1 / 10 \\
\text { nephropathy: } 3 / 10 \text {, micro albumin urea: } 11.3(4.2-46.9)\end{array}$ \\
\hline Endogenous insulin secretion $(n=8)$ & $\begin{array}{l}\text { serum c-peptide immunoreactivity }(\mathrm{ng} / \mathrm{mL}): 0.29(0.06-0.43) \\
\text { c peptide index: } 0.10(0.03-0.21)\end{array}$ \\
\hline SGLT2-I $(n)$ & Ipragliflozin $50 \mathrm{mg} /$ day: 6, Dapagliflozin $5 \mathrm{mg} /$ day: 4 \\
\hline Other drugs $(n)$ & $\alpha$-GI: 5, Metformin: 2 \\
\hline \multicolumn{2}{|l|}{ Glucose parameters detected by isCGM } \\
\hline SD of glucose (mg/dL) & $69.0(52.8-76.0)$ \\
\hline Average plasma glucose $(\mathrm{mg} / \mathrm{L})$ & $184.0(174.2-236.5)$ \\
\hline$\% \mathrm{CV}$ & $34.3(23.3-44.2)$ \\
\hline Percent time with glucose at $<70 \mathrm{mg} / \mathrm{dL}(\%)$ & $0.5(0-8.1)$ \\
\hline Percent time with glucose at $70-180 \mathrm{mg} / \mathrm{dL}(\%)$ & $39.0(18.0-59.7)$ \\
\hline Percent time with glucose at $>180 \mathrm{mg} / \mathrm{dL}(\%)$ & $56.8(37.8-71.1)$ \\
\hline Maximum plasma glucose (mg/dL) & $310.5(288.3-441.8)$ \\
\hline Minimum plasma glucose (mg/dL) & $73.5(49.0-130.5)$ \\
\hline LBGI & $2.5(0.04-6.7)$ \\
\hline HBGI & $16.1(11.5-23.1)$ \\
\hline
\end{tabular}

Data are median or number (25-75\% values).

HbAlc, hemoglobin A1c; FPG, fasting plasma glucose; AST, aspartate aminotransferase; ALT, alanine aminotransferase; $\gamma$-GTP, gamma-glutamyl transpeptidase; eGFR, estimated glomerular filtration rate; SGLT2-I, sodium-glucose cotransporter-2 inhibitor; $\alpha$-GI, alpha-glucosidase inhibitor; CV, coefficient of variation; LBGI, low blood glucose index; HBGI, high blood glucose index.

years, and 4 were males. The median duration of diabetes was 5.0 years. Participants were mildly obese, with a median BMI of $22.7 \mathrm{~kg} / \mathrm{m}^{2}$. Renal function was normal, with eGFR of $77.7 \mathrm{~mL} / \mathrm{min} / 1.73 \mathrm{~m}^{2}$. Laboratory tests showed poor blood glucose control, with median $\mathrm{HbAlc}$ level at $9.1 \%$. The median dose of total insulin was 34.0 $\mathrm{u}$ /day (bolus insulin $16.0 \mathrm{u} /$ day and basal insulin $17.0 \mathrm{u} /$ day), while 5 patients used alpha-glucosidase inhibitor, and 2 patients were on metformin. The baseline isCGM data are shown in Table 1. 


\section{Changes in glucose parameters detected by isCGM}

Fig. 1 shows the mean values of isCGM parameters for all the participants before (Fig. 1A) and after treatment with SGLT2-I for 7 days (Fig. 1B). We also show isCGM data for all the participants at baseline (Supplementary Fig. 1A) and after treatment with SGLT2-I for 7 days (Supplementary Fig. 1B).

As shown in Table 2, SGLT2-I significantly improved TIR from baseline to day 7 , the main outcome measure $(p=0.005)$. The improvement was significant from day 5 based on the Dunnett test. SGLT2-I also significantly improved the secondary outcome measures, including SD of glucose, average plasma glucose, TAR, maximum plasma glucose, HBGI and average nocturnal plasma glucose (0:00-05:59) from baseline to day 7 (Table 2). In contrast, SGLT2-I had no effect on \%CV, TBR, minimum plasma glucose, LBGI and SD of nocturnal glucose (0:00-05:59) from baseline to day 7 (Table 2).
Univariate analysis of factors affecting the change in the TIR on day 7 showed a significant negative relationship with the TIR at baseline (Table 3). Comparison of patients with and without TIR of $70 \%$ or higher on day 7 showed significant differences in eGFR, baseline mean plasma glucose, baseline maximum plasma glucose, and baseline HBGI between the two groups (Table 4).

\section{Safety}

No serious side effects were observed during the study period. After the addition of SGLT2-I, many patients developed hypoglycemia, as recorded by the isCGM, but there were no significant changes in TBR and minimum plasma glucose (Table 2). Furthermore, none of the patients developed severe hypoglycemia associated with disturbance of consciousness or requiring medical intervention. Also, none of the patients developed ketosis or ketoacidosis after the addition of SGLT2-I.

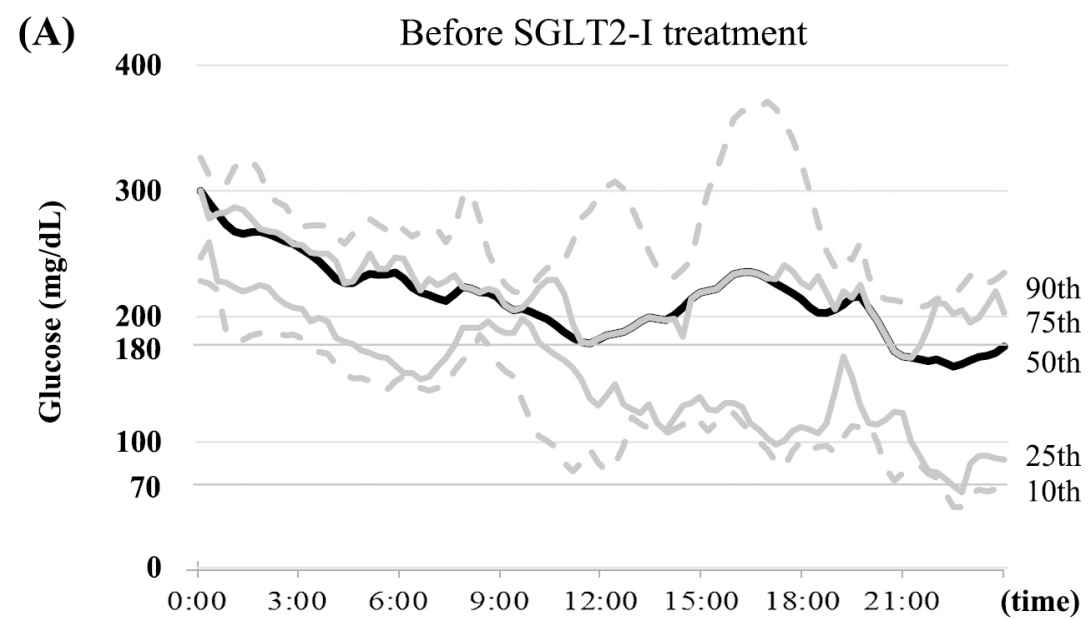

(B) Day 7 after SGLT2-I treatment

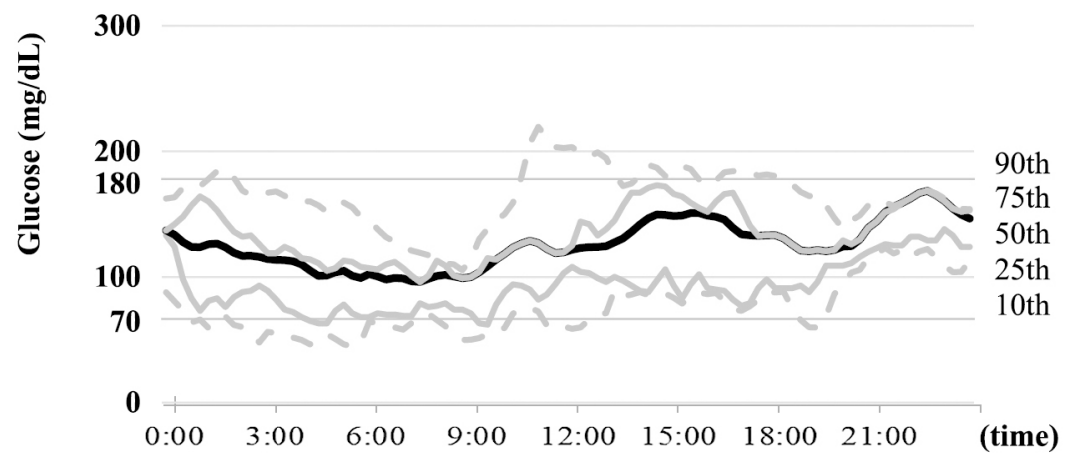

Fig. 1 (A) isCGM data before the addition of SGLT2-I, (B) isCGM data at day 7 after the addition of SGLT2-I. The center solid line is the median, the next outer solid lines are the interquartiles (25th and 75th percentiles), and the dotted lines depict the 10th and 90th percentiles. isCGM, flash glucose monitoring; SGLT2-I, sodium-glucose cotransporter 2 inhibitor. 


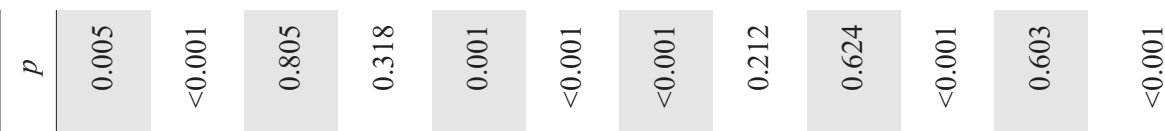

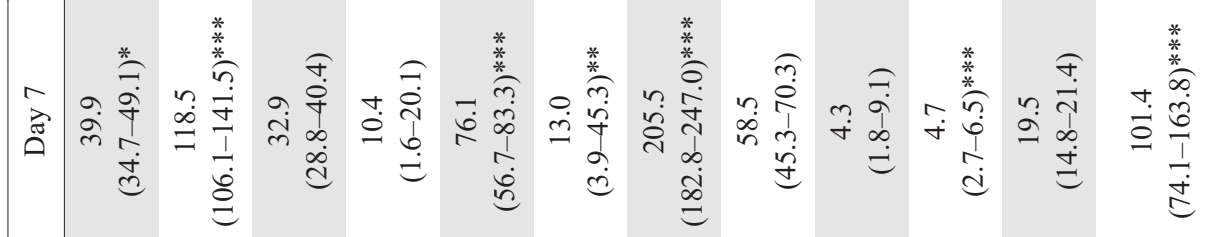

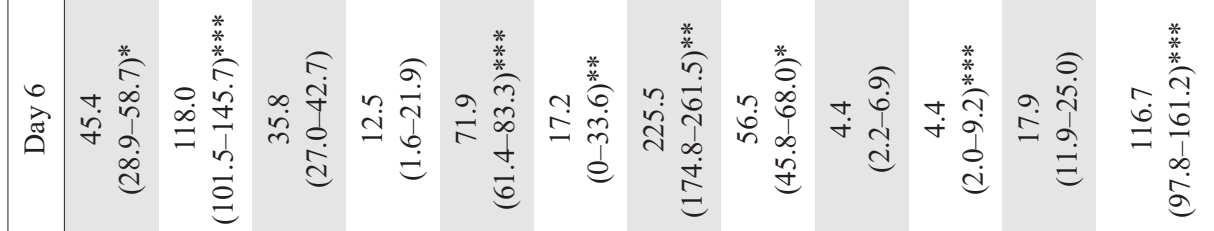

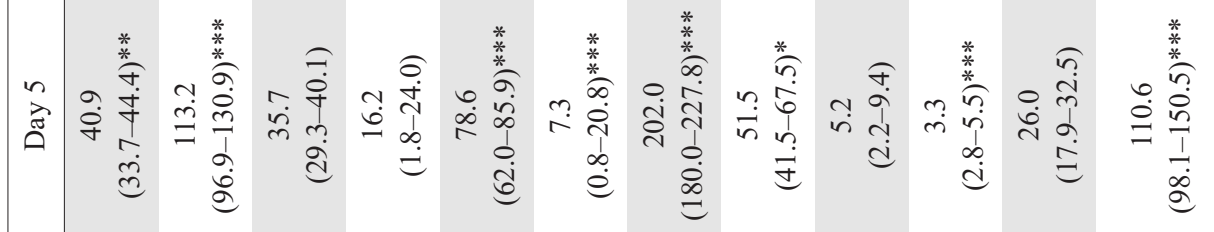

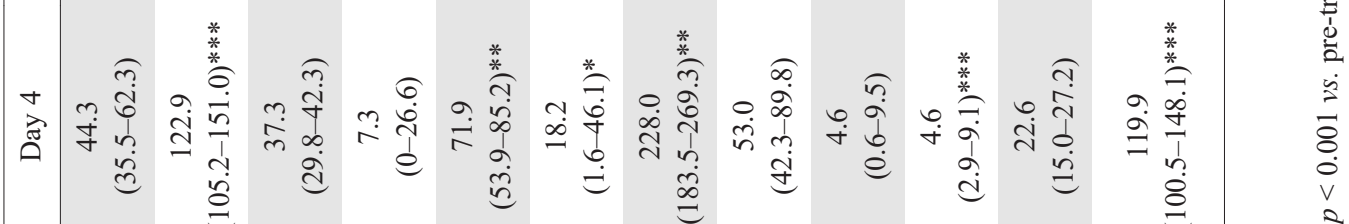

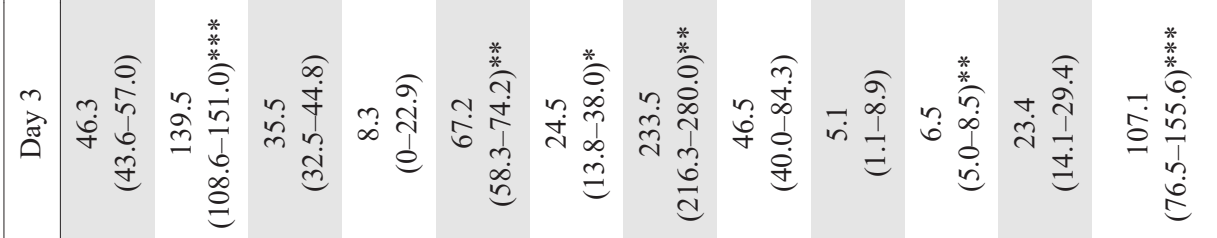

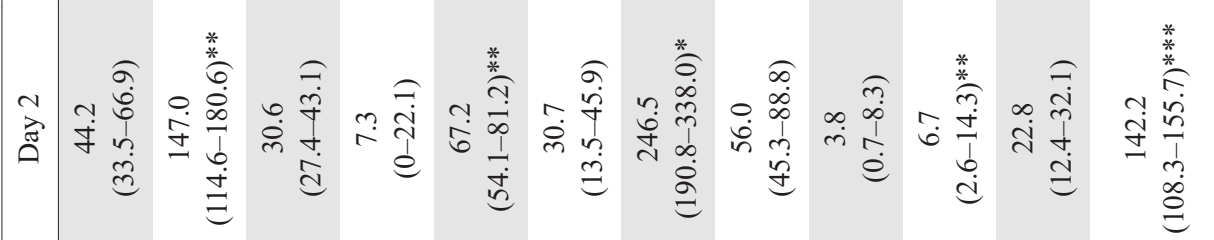

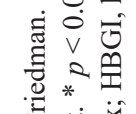

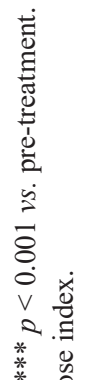

क.

$\checkmark \frac{8}{*}$

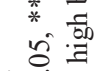

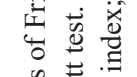

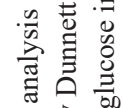

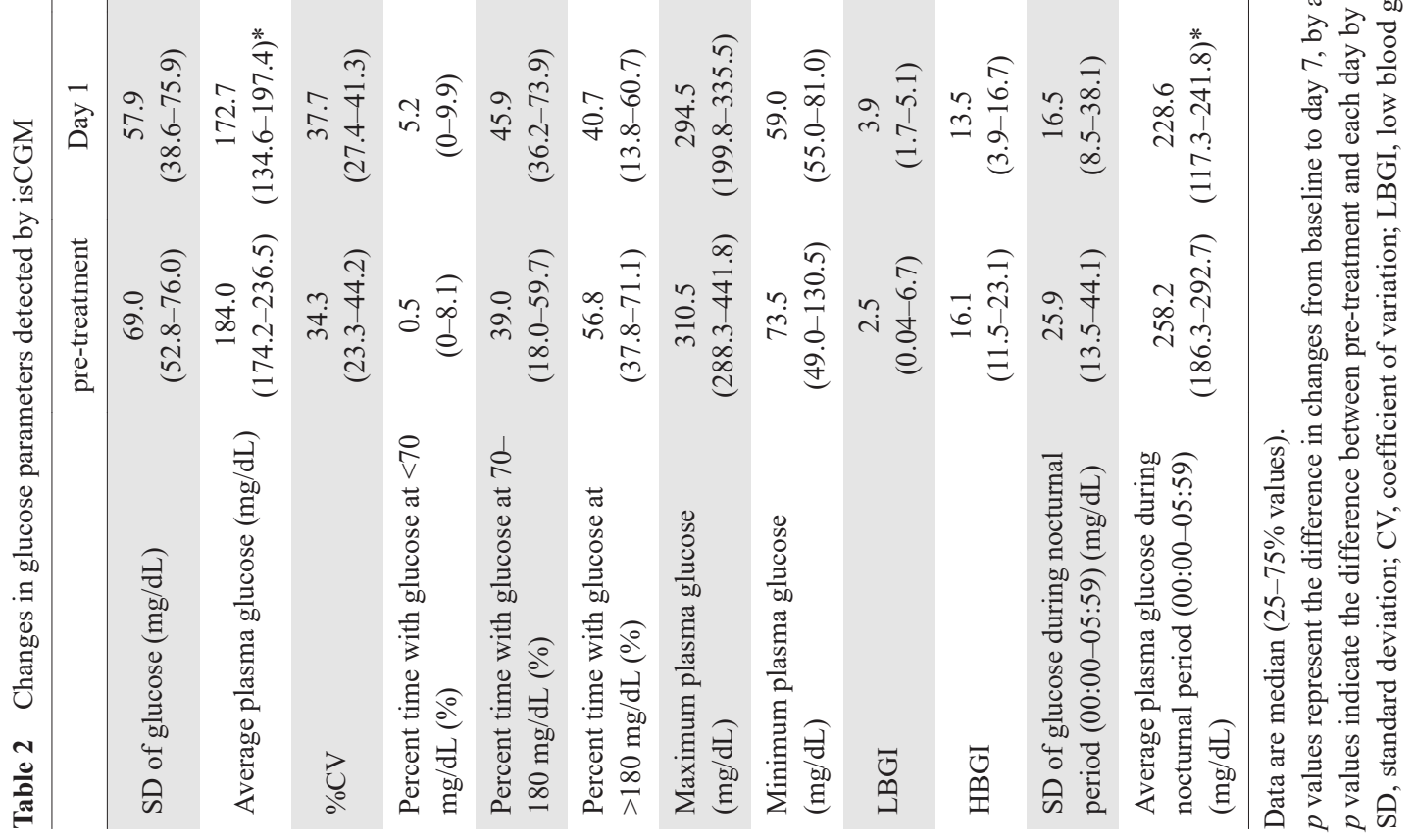




\section{Discussion}

The results of the present study using isCGM in an actual clinical setting for the treatment of patients with T1DM demonstrated that the combination of SGLT2-I and intensive insulin therapy improved plasma glucose fluctuations and mean plasma glucose levels and at the

Table 3 Univariate linear regression analysis for predicting change of percent time with glucose at $70-180 \mathrm{mg} / \mathrm{dL}$ on day 7

\begin{tabular}{lcc}
\hline \multirow{2}{*}{ Variable } & \multicolumn{2}{c}{ Univariate Analysis } \\
\cline { 2 - 3 } & $r$ & $p$ value \\
\hline Age & 0.122 & 0.738 \\
Diabetes duration & 0.621 & 0.055 \\
Body mass index & 0.238 & 0.509 \\
HbA1c & -0.309 & 0.385 \\
eGFR & 0.017 & 0.963 \\
Total required insulin dose & 0.197 & 0.585 \\
Percent time with glucose at $70-180$ & -0.706 & 0.023 \\
mg/dL at baseline & & \\
\hline
\end{tabular}

Data are $p$-value by Spearman rank correlation.

$\mathrm{HbA1c}$, hemoglobin A1c; eGFR, estimated glomerular filtration rate same time did not worsen hypoglycemic indices.

In the present study, the TIR, the primary endpoint, increased from $39.0 \%$ before the use of SGLT2-I to $76.1 \%$ on treatment day 7 . Although none of the patients achieved a TIR of $70 \%$ or higher before the addition of SGLT2-I, six patients met that TIR criterion on treatment day 7. The SD of glucose, average plasma glucose, TAR, maximum plasma glucose and average nocturnal plasma glucose (i.e., the secondary endpoints) also improved significantly following the addition of SGLT2-I (Table 2). In contrast, no significant changes were observed in various hypoglycemic indices used in this study, such as the TBR and minimum plasma glucose (similar results for the nocturnal-related parameters). The magnitude of the change in TIR on day 7 correlated significantly with the baseline TIR (Table 3). These results indicate that short-term treatment with SGLT2-I resulted in a larger improvement in TIR in patients with poor glycemic control with lower baseline TIR. Analysis of data of patients with and without TIR of $\geq 70 \%$ on day 7 also showed significant differences in eGFR, average baseline plasma glucose, baseline maximum plasma glucose, and baseline HBGI between the two groups (Table 4). Based on these results, we conclude that patients with better glycemic control at baseline are more likely to achieve a TIR of

Table 4 Differences between achieved group and non-achieved group (Percent time with glucose at $70-180 \mathrm{mg} / \mathrm{dL} \geq 70 \%$ )

\begin{tabular}{|c|c|c|c|}
\hline & achieved group $(n=6)$ & Non-achieved group $(n=4)$ & $p$ value \\
\hline Age (year) & $55.0(40.5-61.5)$ & $62.0(46.3-62.8)$ & 0.392 \\
\hline Diabetes duration (year) & $5.0(1.8-10.8)$ & $5.5(2.3-8.0)$ & 0.914 \\
\hline Body mass index $\left(\mathrm{kg} / \mathrm{m}^{2}\right)$ & $22.6(20.6-25.6)$ & $24.7(21.3-27.6)$ & 0.670 \\
\hline HbA1c (\%) & $9.1(9.0-9.7)$ & $8.9(8.7-9.0)$ & 0.065 \\
\hline eGFR $\left(\mathrm{mL} / \mathrm{min} / 1.73 \mathrm{~m}^{2}\right)$ & $86.5(78.2-91.2)$ & $69.2(65.1-74.7)$ & 0.019 \\
\hline Required insulin dose (u/day) & $32.0(19.5-58.5)$ & $42.5(26.3-57.3)$ & 0.521 \\
\hline SD of glucose at baseline (mg/dL) & $61.4(47.7-76.0)$ & $69.5(65.8-84.5)$ & 0.286 \\
\hline Average plasma glucose at baseline $(\mathrm{mg} / \mathrm{dL})$ & $175.8(166.7-190.6)$ & $267.6(186.7-351.6)$ & 0.033 \\
\hline$\% \mathrm{CV}$ at baseline & $35.8(25.0-44.2)$ & $26.7(19.5-45.6)$ & 0.476 \\
\hline Percent time with glucose at $<70 \mathrm{mg} / \mathrm{dL}$ at baseline (\%) & $2.1(0-9.1)$ & $0(0-10.1)$ & 0.363 \\
\hline Percent time with glucose at $70-180 \mathrm{mg} / \mathrm{dL}$ at baseline (\%) & $50.5(34.9-60.7)$ & $17.7(0-38.5)$ & 0.054 \\
\hline Percent time with glucose at $>180 \mathrm{mg} / \mathrm{dL}$ at baseline (\%) & $47.4(37.2-59.1)$ & $80.2(41.7-100.0)$ & 0.199 \\
\hline Maximum plasma glucose at baseline (mg/dL) & $291.5(278.0-317.0)$ & $451.5(343.5-488.3)$ & 0.019 \\
\hline Minimum plasma glucose at baseline (mg/dL) & $60.5(49.0-93.5)$ & $140.0(49.8-201.8)$ & 0.392 \\
\hline LBGI at baseline & $3.8(0.5-6.7)$ & $0.7(0-9.6)$ & 0.476 \\
\hline HBGI at baseline & $12.7(8.8-15.8)$ & $29.0(16.9-45.9)$ & 0.019 \\
\hline
\end{tabular}

Data are median (25-75\% values).

$p$ values for comparison of the achieved and non-achieved groups, by Mann-Whitney $U$ test.

HbA1c, hemoglobin A1c; eGFR, estimated glomerular filtration rate; SD, standard deviation; CV, coefficient of variation; LBGI, low blood glucose index; HBGI, high blood glucose index. 
$\geq 70 \%$ on day 7 of treatment with SGLT2-I. The results of the present study are important because they demonstrated in real clinical setting that the addition of SGLT2I improved the TIR without increasing the incidence of hypoglycemia in patients with T1DM. Although studies using isCGM to assess the effects of SGLT-I on T1DM have been sporadically reported in western countries, the present pilot study is the first to be conducted in an actual clinical setting in Japan.

With regard to the efficacy of SGLT-I in T1DM, Kaku et al. [8] reported that the concomitant use of ipragliflozin with insulin significantly improved $\mathrm{HbA} 1 \mathrm{c}$ levels $(-0.47 \%$, versus placebo: $-0.36 \%)$, reduced body weight $(-2.92 \mathrm{~kg}$, versus placebo: $-2.87 \mathrm{~kg})$, and reduced total daily insulin dose $(-6.64 \mathrm{u} /$ day, versus placebo: $-7.35 \mathrm{u} /$ day). These findings confirmed the similar and earlier results of Mathieu et al. [9]. In a study using CGM to evaluate the efficacy of SGLT2-I in patients with T1DM, Rodbard et al. [10] reported that the concomitant use of canagliflozin (100 mg or $300 \mathrm{mg}$ ) through monitoring by Dexcom G4 Platinum increased the TIR (100 mg: $+11.6 \%, 300 \mathrm{mg}:+10.1 \%)$ and decreased the TAR (100 mg: $-12.7 \%, 300 \mathrm{mg}:-7.6 \%$ ) without increasing the TBR in patients with T1DM. Similar findings were reported more recently by others $[11,12]$. While the present study also yielded similar results, improvement in nocturnal plasma glucose levels has not been reported previously. This finding suggested that SGLT2-I might be especially suited for patients in whom larger insulin doses should be avoided in order to reduce the risk of hypoglycemia. While the present study did not measure glucagon levels, SGLT2-I reportedly promotes glucagon production $[13,14]$, which might be one of the mechanisms by which it prevents hypoglycemia. In addition, in our previous study [15], which used CGM to assess the effects of SGLT2-I on T2DM, the incidence of hypoglycemia did not increase after 1 week of treatment, and the lowest plasma glucose level at 1 week correlated well with urinary glucose excretion. The results of that study demonstrated substantial inhibition of urinary glucose excretion in patients with the lowest plasma glucose levels.

With regard to the adverse effects of treatment, our results showed no incidence of severe hypoglycemia, the most concerning hypoglycemia-associated event. In the above-mentioned meta-analysis of 14 randomized controlled trials, the incidence of adverse events was comparable between SGLT2-I and placebo, and no significant increase in the incidence of severe hypoglycemia was observed [4]. As described above, the DEPICT-1/ DEPICT-2 trial showed that dapagliflozin did not signifi- cantly increase the TBR compared with placebo [11].

Clinical trials conducted in Japan and other countries have reported that the use of SGLT2-I for T1DM is associated with the risk of diabetic ketoacidosis (DKA) [8, 9, 16, 17]. SGLT2-I increases glucagon production through urinary glucose excretion and its direct action on pancreatic $\alpha$ cells [18]. In turn, glucagon enhances lipolysis and ketogenesis [19]. Decreased sodium reabsorption in the kidney may inhibit ketone body excretion [20]. DKA appears to occur through these mechanisms. In a metaanalysis study, Goldenberg et al. [21] reported that the addition of SGLT2-I to the treatment of T1DM increased the risk of DKA by 3.47-fold. In another meta-analysis study, Masri et al. [22] also reported that the concomitant use of SGLT2-I for T1DM increased the risk of DKA but did not increase significantly the incidence of other adverse events; hypoglycemia or infection. None of the patients in the present study experienced severe hypoglycemia or ketosis.

The limitations of the present study are: (1) the relatively small sample size of 10 patients, (2) the retrospective study design, (3) the lack of a control group, and (4) the lack of consistency in meal amounts and times due to the conduct of the study in an outpatient setting. We plan in the future to conduct a prospective study with a larger sample size in an inpatient setting.

In conclusion, we have demonstrated in the present study using the isCGM in an actual clinical setting that the addition of SGLT2-I to intensive insulin therapy did not increase the incidence of hypoglycemia and rather improved plasma glucose fluctuations and mean plasma glucose levels in patients with T1DM. Our results suggest that the use of SGLT2-Is in the indicated T1DM patients under careful attention to potential risks, can significantly improve the TIR. Such therapeutic protocol can easily achieve TIR of $\geq 70 \%$, which is the recommended target for diabetes therapy recently adopted by consensus.

\section{Acknowledgments}

We thank the study participants.

\section{Disclosure}

Y.O. had received consultancy fees from Astellas, MSD and Ono Pharm Corporation. Y.T. has received speaking fees and/or honoraria from Astellas. All other authors declare no conflict of interest. 


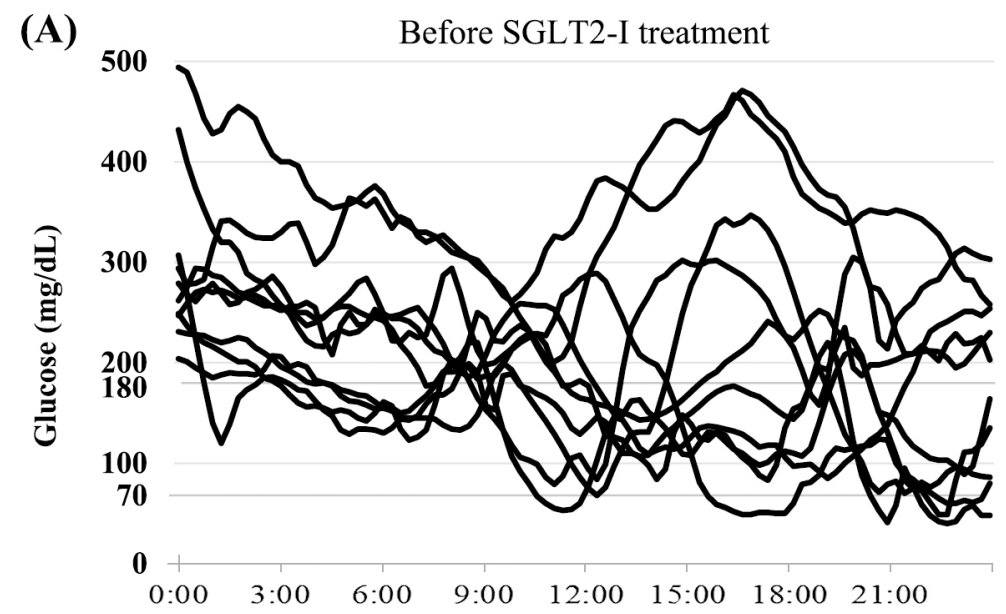

(B) $500 \quad$ Day 7 after SGLT2-I treatment

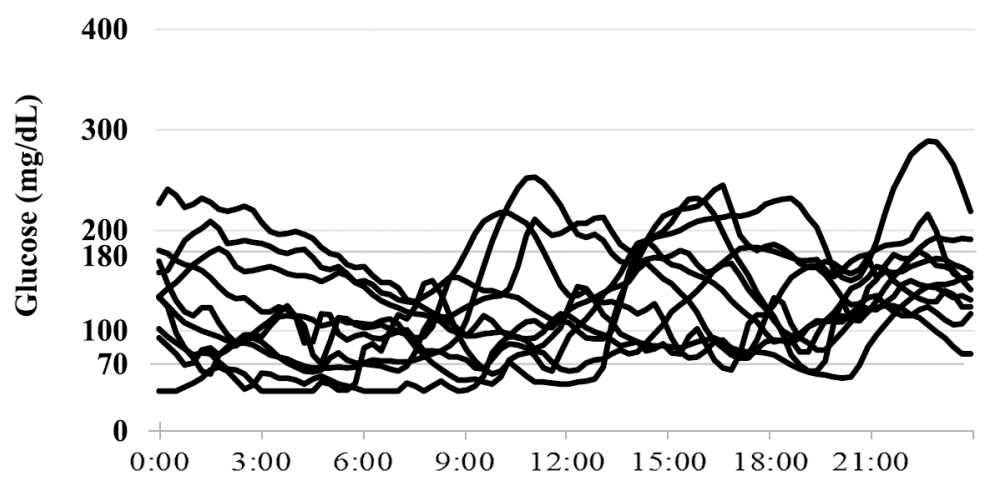

Supplementary Fig. 1 24-hr blood glucose levels of the 10 study patients, as measured by the isCGM, at (A) baseline and (B) after 7 days of treatment with insulin combined with SGLT2-I.

\section{References}

1. Dandona P, Mathieu C, Phillip M, Hansen L, Griffen SC, et al. (2017) Efficacy and safety of dapagliflozin in patients with inadequately controlled type 1 diabetes (DEPICT-1): 24 week results from a multicentre, doubleblind, phase 3, randomised controlled trial. Lancet Diabetes Endocrinol 5: 864-876.

2. Rosenstock J, Marquard J, Laffel LM, Neubacher D, Kaspers S, et al. (2018) Empagliflozin as adjunctive to insulin therapy in type 1 diabetes: the EASE trials. Diabetes Care 41: 2560-2569.

3. Garg SK, Henry RR, Banks P, Buse JB, Davies MJ, et al. (2017) Effects of sotagliflozin added to insulin in patients with type 1 diabetes. N Engl J Med 377: 2337-2348.

4. Yamada T, Shojima N, Noma H, Yamauchi T, Kadowaki $T$ (2018) Sodium-glucose co-transporter-2 inhibitors as add-on therapy to insulin for type 1 diabetes mellitus: systematic review and meta-analysis of randomized controlled trials. Diabetes Obes Metab 20: 1755-1761.

5. Battelino T, Danne T, Bergenstal RM, Amiel SA, Beck R, et al. (2019) Clinical targets for continuous glucose moni- toring data interpretation: recommendations from the international consensus on time in range. Diabetes Care 42: 1593-1603.

6. Lu J, Ma X, Zhou J, Zhang L, Mo Y, et al. (2018) Association of time in range, as assessed by continuous glucose monitoring, with diabetic retinopathy in type 2 diabetes. Diabetes Care 41: 2370-2376.

7. Viegersky RA, McMahon C (2019) The relationship of hemoglobin A1C to time-in-range as an outcome measure for diabetes. Diabetes Technol Ther 21: 81-85.

8. Kaku K, Isaka H, Sakatani T, Toyoshima J (2019) Efficacy and safety of ipragliflozin add-on therapy to insulin in Japanese patients with type 1 diabetes mellitus: a randomized, double-blind, phase 3 trial. Diabetes Obes Metab 21: 2284-2293.

9. Mathieu C, Dandona P, Gillard P, Senior P, Hasslacher C, et al. (2018) Efficacy and safety of dapagliflozin in patients with inadequately controlled type 1 diabetes (the DEPICT-2 study): 24-week results from a randomized controlled trial. Diabetes Care 41: 1938-1946. 
10. Rodbard HW, Peters AL, Slee A, Cao A, Traina SB, et al. (2017) The effect of canagliflozin, a sodium glucose cotransporter 2 inhibitor, on glycemic end points assessed by continuous glucose monitoring and patient-reported outcomes among people with type 1 diabetes. Diabetes Care 40: 171-180.

11. Mathieu C, Dandona P, Phillip M, Oron T, Lind M, et al. (2019) Glucose variables in type 1 diabetes studies with dapagliflozin: pooled analysis of continuous glucose monitoring data from DEPICT-1 and -2. Diabetes Care 42: 1081-1087.

12. Danne T, Cariou B, Buse JB, Garg SK, Rosenstock J, et al. (2019) Improved time in range and glycemic variability with sotagliflozin in combination with insulin in adults with type 1 diabetes: a pooled analysis of 24-week continuous glucose monitoring data from the in tandem program. Diabetes Care 42: 919-930.

13. Merovci A, Solis-Herrera C, Daniele G, Eldor R, Fiorentino TV, et al. (2014) Dapagliflozin improves muscle insulin sensitivity but enhances endogenous glucose production. J Clin Invest 124: 509-514.

14. Ferrannini E, Muscelli E, Frascerra S, Baldi S, Mari A, et al. (2014) Metabolic response to sodium-glucose cotransporter 2 inhibition in type 2 diabetic patients. J Clin Invest 124: 499-508.

15. Torimoto K, Okada Y, Koikawa K, Tanaka Y (2017) Early effects of sodium-glucose co-transporter 2 inhibitors in type 2 diabetes: study based on continuous glucose monitoring. Diabetol Metab Syndr 9: 60.
16. Araki E, Watada H, Uchigata Y, Tomonaga O, Fujii H, et al. (2020) Efficacy and safety of dapagliflozin in Japanese patients with inadequately controlled type 1 diabetes (DEPICT-5): 52-week results from a randomized, openlabel, phase III clinical trial. Diabetes Obes Metab 22: 540-548.

17. McCrimmon RJ, Henry RR (2018) SGLT inhibitor adjunct therapy in type 1 diabetes. Diabetologia 61: 2126-2133.

18. Bonner C, Kerr-Conte J, Gmyr V, Queniat G, Moerman E, et al. (2015) Inhibition of the glucose transporter SGLT2 with dapagliflozin in pancreatic alpha cells triggers glucagon secretion. Nat Med 21: 512-517.

19. Liljenquist JE, Bomboy JD, Lewis SB, Sinclair-Smith BC, Felts PW, et al. (1974) Effects of glucagon on lipolysis and ketogenesis in normal and diabetic men. J Clin Invest 53: 190-197.

20. Taylor SI, Blau JE, Rother KI (2015) SGLT2 inhibitors may predispose to ketoacidosis. $J$ Clin Endocrinol Metab 100: 2849-2852.

21. Goldenberg RM, Gilbert JD, Hramiak IM, Woo VC, Zinman B (2019) Sodium-glucose co-transporter inhibitors, their role in type 1 diabetes treatment and a risk mitigation strategy for preventing diabetic ketoacidosis: the STOP DKA protocol. Diabetes Obes Metab 21: 21922202.

22. El Masri D, Ghosh S, Jaber LA (2018) Safety and efficacy of sodium-glucose cotransporter 2 (SGLT2) inhibitors in type 1 diabetes: a systematic review and meta-analysis. Diabetes Res Clin Pract 137: 83-92. 